\title{
ANÁLISIS DE TENDENCIAS EN LA ENSEÑANZA DE POSGRADO EN DISEÑO EN UN CONTEXTO DE DIVERSIFICACIÓN DE LA PROFESIÓN DEL DISEÑADOR ${ }^{*}$
}

\author{
Magdalena Vicuña ${ }^{1}$
}

\section{RESUMEN}

El presente trabajo consiste en una investigación exploratoria que se basa en la recopilación de antecedentes relevantes que permitan entender las tendencias en los diversos ámbitos de enseñanza de la disciplina del Diseño a nivel de Magíster. Se analizó un total de 39 programas de Magíster, pertenecientes a 25 Escuelas de Diseño del mundo, con base en lo señalado en páginas web y documentos solicitados en relación con su presentación, enfoque, y respectiva malla curricular.

Entre las principales tendencias encontradas en los programas de magíster en Diseño seleccionados, independientemente de su área de especialización, se encuentran: (1) Énfasis en la práctica y el vínculo con la realidad; (2) Multidisciplinareidad; (3) El usuario como centro del proyecto de diseño; (4) Importancia de la investigación aplicada al proyecto de diseño, y (5) El creciente rol del diseño en la estrategia y gestión de empresas.

Palabras clave: tendencias, educación superior de posgrado, diseño.

\section{ANALYSIS OF TRENDS IN POSTGRADUATE DESIGN TEACHING IN A CONTEXT OF DIVERSIFICATION OF THE DESIGN PROFESSION}

\section{ABSTRACT}

This article presents findings of a research based on the collection of evidence which allow understanding trends having place in diverse areas of design teaching at a master degree. The research has analyzed a total of 39 Masters Programs belonging to 25 Schools of Design based on their websites and requested documents related to statements, focus and course structures.

Regardless of the area of specialization, among the main trends found in selected Masters Degree Programs it can be highlighted: (1) focus on practice and connection with the public and private sector; (2) multidisciplinary context; (3) users as the core of a design project; (4) importance of the applied research to the design project and (5) an increasing role of design in business strategies and management.

Key words: trends, graduate education, design.

1 Escuela de Diseño, Pontificia Universidad Católica de Chile. Chile. Contacto: mvicunad@uc.cl

* Colaboradores: Carola Zurob, diseñadora; Alberto González, Álvaro Sylleros, profesores asociados. 


\section{ANÁLISIS DE TENDENCIAS EN LA ENSEÑANZA DE POSGRADO EN DISEÑO EN UN CONTEXTO DE DIVERSIFICACIÓN DE LA PROFESIÓN DEL DISEÑADOR}

\section{Introducción}

El presente trabajo consiste en una investigación de carácter exploratorio que se basa en la recopilación de antecedentes relevantes que permitan entender los diversos ámbitos de enseñanza de la disciplina del diseño en el grado de magíster. Esta investigación se realiza en el contexto de la creación de un nuevo proyecto de magíster en la Escuela de Diseño de la Pontificia Universidad Católica de Chile (PUC), con la finalidad de focalizar los contenidos de la enseñanza de posgrado a las demandas reales de especialización de diseñadores y profesionales relacionados al diseño, en el ámbito nacional e internacional.

La Escuela de Diseño de la PUC ha desarrollado sus políticas académicas bajo premisas que se fundamentan desde una definición conceptual de la disciplina, que coloca la relevancia en el proceso de diseño y en todo lo referido al ciclo de vida del producto diseñado. Así, se ha persistido en que el trinomio proyecto-producto-persona sea resguardado desde una visión integrada y transversal, tal como se plantea en el Plan de Desarrollo de la Escuela de Diseño: "la Escuela de Diseño de la Pontificia Universidad Católica de Chile entiende el diseño como una actividad creativa y de producción al servicio de los demás, centrada en la interacción entre el ser humano y su entorno; esto es, desde la observación de la interacción entre identidades, se diseña un producto cuya calidad es testeada, para asegurar máximas prestaciones en diseño y usabilidad, con la consiguiente rentabilidad en el mercado. Para la consecución de este objetivo se requiere el estudio del usuario y su conocimiento, como garante de toda interacción factible entre lo artificial, el sujeto y su entorno."

Así, el proyecto educativo de la Escuela se orienta a desarrollar el pensamiento interdisciplinario y humanista en sus estudiantes, 
promoviendo su espíritu crítico e innovador, desde una visión estratégica del mundo y conciencia social, contribuyendo a la innovación, desarrollo y beneficio social de Chile. ${ }^{2}$

Lejos de referirse a una mera formalización de un concepto o idea, para la Escuela de Diseño, el proyecto constituye la consolidación de la misma en una obra, reconocible y capaz de generar interacciones con un usuario. En dicha relación el objeto cobra un significado basado en su funcionalidad y utilidad, medible en la interacción objeto y persona. Es por esto que junto a dicha preocupación por los procesos de producción del diseño, se investiga también al usuario/ consumidor como generador tanto de una oportunidad de diseño, como de directrices formales.

A su vez, se reconoce que este resultado es también generador de emociones que ponen al sujeto ante límites allegados a las artes mayores. Conscientes de esta relación compleja, la representación material del proyecto es de gran relevancia, abordando esta materia desde todas las plataformas encontradas en el arte y la tecnología.

Durante el último decenio, la disciplina del diseño ha sido tensionada por requerimientos de tipo económico, social, medioambiental y tecnológico, los cuales hacen del proyecto de diseño un proyecto de alta complejidad. Sin renegar de su origen estético formal, el estudio de los elementos que condicionan la morfología del proyecto se ha expandido en forma significativa. En efecto, los mercados globales crecientemente demandan a los profesionales del diseño competencias tendientes a vincular los procesos proyectuales propios del diseño con las necesidades de gestión del producto, abarcando todo su ciclo de vida, desde la ideación, industrialización, seriación, comercialización, hasta su experiencia de compra y uso. También corresponden al proyecto las preocupaciones medioambientales relativas tanto al uso de recursos naturales, industriales y tecnológicos, como de la obsolescencia y reciclado de los desechos que genera el uso de un determinado sistema o producto.

2 Plan de Desarrollo 2006-2010, Escuela de Diseño, Pontificia Universidad Católica de Chile. 
En este sentido, la educación formal en diseño, ya sea de pregrado o posgrado, constituye el contexto fundamental para la promoción y apoyo al diseño por parte del sector público, en especial para las pequeñas y medianas empresas, ya que es justamente en estas empresas en las cuales se requiere de profesionales con conocimientos de diseño estratégico y de gestión. Así, la formación de buenos profesionales del diseño es necesaria para satisfacer la demanda por diseño que pueda surgir a partir del éxito de dichas políticas de incentivos. $^{3}$

El proceso de diseño también debe incorporar metódicamente el máximo de variables surgidas desde la comprensión de la interacción sujeto-objeto, relacionadas con la identidad personal, colectiva, narrativa y cognitiva en que se inserta el proyecto.

Todos los elementos mencionados han generado una creciente complejización en la aproximación al proyecto de diseño, el cual requiere necesariamente de un proceso de pensamiento crítico y sistémico, con la finalidad de conceptualizar, aplicar, analizar, sintetizar y/o evaluar de manera efectiva, la información generada a partir de la observación, la experiencia, la reflexión, el razonamiento y la comunicación. En lo fundamental, el proceso de diseño implica el ejercicio de anticipar, de proyectar pensando en un proceso dinámico, más que en lograr un resultado indiferente fruto de una proyección lineal (ver figura n. ${ }^{\circ} 1$ ). Así, se incorpora la variable creativa desde una mirada metodológica e instrumental para lograr un proceso de innovación sistémico.

Figura $n .^{\circ} 1$

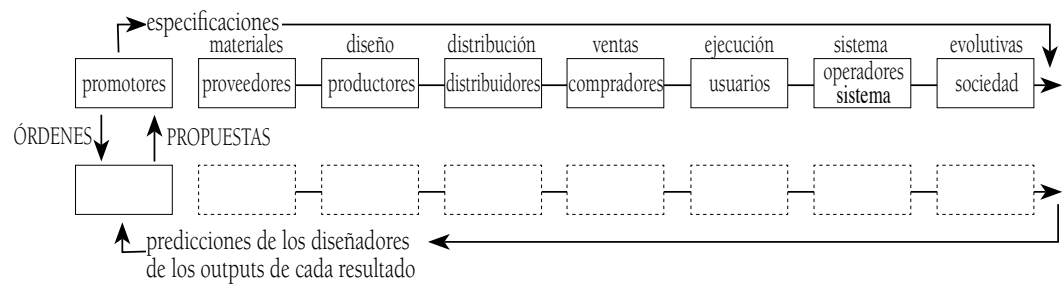

Fuente: Jones (1984).

3 Ver Raulik-Murphy, Cawood, Larsen y Lewis (2008). 
En este contexto de diversificación de la profesión del diseñador y ante la necesidad de formar no sólo profesionales especializados ${ }^{4}$, sino que también producir investigación en diseño orientada al desarrollo del país, cabe preguntarse entonces: ise refleja esta complejidad del proyecto en la enseñanza de posgrado en diseño en el ámbito internacional? y icuáles son los principales aspectos relativos a la complejidad del proyecto de diseño en los cuales se enfoca la enseñanza de posgrado en diseño?

Es posible suponer que la creciente complejización en la aproximación al proyecto de diseño está siendo abordada por los programas de magíster en el mundo, desde sus respectivas especificidades y campos de acción. De esta manera, esta investigación pretende definir lineamientos y tendencias sobre los principales enfoques de los programas de posgrado, específicamente programas de magíster de diseño.

Los objetivos de esta investigación se enumeran a continuación:

- Hacer un registro de programas que refleje las principales tendencias de la educación de posgrado de diseño en el mundo, en países desarrollados de occidente.

- Identificar las áreas de estudio más importantes que imparten los programas de posgrado en diseño seleccionados.

- Identificar el enfoque de la disciplina que establecen aquellos programas de posgrado en diseño de orientación integral a la disciplina.

- Determinar el tipo de diseñador que se perfila a partir de las tendencias identificadas en la educación de posgrado de diseño.

- Identificar nuevas áreas estratégicas para la elaboración de un futuro magíster en diseño.

4 A la fecha, en Chile existen sólo seis programas de magíster en diseño dirigidos a diseñadores o profesionales de áreas afines. Esta incipiente oferta nacional de posgrados en diseño se caracteriza por la orientación hacia prácticas proyectuales específicas, basados en el estudio de ciertas especialidades del diseño, algunos de ellos orientados directamente hacia las necesidades de la industria nacional. 


\section{Metodología utilizada}

La metodología utilizada para la realización del presente trabajo se basó en el análisis de contenido de la información disponible en los sitios web de los programas seleccionados, además de información adicional solicitada vía correo electrónico a las distintas instituciones.

Una primera aproximación para seleccionar los programas de magíster en diseño para analizar se realizó a través de la consulta a diversos expertos en la materia, quienes sugirieron un total de 60 programas. Los expertos consultados, Prasad Boradkar (Chair Person at Arizona State University), Nathan Shedroff (California College of Arts) y Alex Blanch (ex director Escuela de Diseño PUC y ex subdirector y coordinador del Proyecto Educativo y jefe de Relaciones Internacionales de Escola Superior de Disseny, Elisava, Barcelona), constituyen destacados académicos y directivos en el ámbito del diseño. Para esta selección se consideraron tanto los programas tradicionales de posgrado en diseño, como aquellos programas con innovaciones temáticas, considerando especialmente los que se desarrollan en países similares a Chile en cuanto a su geografía, estructura económica y cultura.

Al primer listado sugerido por los expertos anteriormente mencionados, se sumaron los programas de magíster seleccionados por exalumnos y académicos de la Escuela de Diseño de la PUC para cursar estudios de posgrado. Posteriormente, como tercer criterio de selección, se agregaron los programas pertenecientes a las 50 mejores escuelas de diseño del mundo, de acuerdo con el ranking realizado por la revista Business Week (2007) ${ }^{5}$, más los programas mencionados en el ranking de la revista Print (2009), relacionados principalmente con el ámbito gráfico.

No se consideraron programas en Latinoamérica, ya que, o no estaban presentes en los rankings mencionados, o no publicaban información suficiente para su análisis. Tampoco se consideraron

5 Ver: http://images.businessweek.com/ss/07/10/1005_dschools/index_01. htm?chan=innovation_special+report+--+d-schools_special+report+--+d-schools, fecha de consulta 20/06/09 
programas en Asia, por constituir esta región materia significativa para futuras investigaciones.

La sumatoria de todos estos programas proporcionó un total de 171 programas de magíster en diversas especialidades del diseño, pertenecientes a un total de 36 instituciones.

Con la finalidad de hacer un estudio profundo de los programas de magíster más afines al ámbito universitario y al proyecto educativo de la Escuela de Diseño, se hizo un primer filtro, eliminando todos aquellos programas que se insertan dentro de institutos y escuelas independientes de Arte y de Diseño. Sobre la base del listado completo de programas se determinó que, en términos generales, los institutos o escuelas independientes tienden a dictar programas más ligados a industrias específicas (como la automotriz o la moda) o al arte y la artesanía. Por lo tanto, para el análisis se privilegiaron los programas dictados por escuelas o institutos pertenecientes a universidades que tuvieran un enfoque más integral del proyecto de diseño. Se mantuvieron de todas maneras los programas dictados por institutos o escuelas independientes que hacen un aporte en términos de la temática que abordan. Por último, se eliminaron todos los programas de doctorado, de manera de dejar sólo los que corresponden al grado de magíster o maestría.

El resultado de este primer filtro arrojó un total de 98 programas de magíster, pertenecientes a 29 escuelas de diseño de todo el mundo.

Posteriormente, se realizó un segundo filtro a través de la selección de aquellos programas que presentan uno o más de los siguientes "atributos deseables": (1) Interdisciplinaridad (determinada por los programas dictados en conjunto con escuelas y/o institutos de disciplinas distintas al diseño); (2) Programas vinculados a la gestión del diseño (Design Management); (3) Programas con un fuerte énfasis en la etapa de investigación dentro del proyecto de diseño o vinculados al Design Research, y (4) Un enfoque de diseño integral. Se mantuvieron también aquellos programas con un enfoque innovador o estratégico para la Escuela de Diseño PUC, que podrían abrir un campo de acción nuevo para el diseño en Chile. 
Este segundo filtro arrojó un total de 39 programas de magíster, pertenecientes a 25 escuelas de diseño, de los cuales se analizó su presentación y su respectiva malla curricular (ver figura n..$^{\circ}$ 2). Los programas identificados tanto en el primer como el segundo filtro se agruparon según las siguientes ocho macroáreas de estudio: Diseño Industrial, Diseño de Productos, Comunicación Visual, Design Research, Diseño de Interacción, Diseño de Espacios y Ambientes, Diseño Textil e Indumentaria, Diseño y Gestión, Teoría, Crítica e Historia del Diseño. Estas macroáreas se desprenden de la propia definición que hacen los programas seleccionados. Se consideran válidas en cuanto reconocen los clásicos ámbitos del desempeño profesional de los diseñadores (Diseño Industrial, Diseño Textil o Diseño de Espacios) y dan cuenta de nuevos campos de acción (gestión, interacción, Design Research), los cuales constituyen ámbitos más interdisciplinarios que abren nuevos espacios en el campo laboral de los diseñadores. 


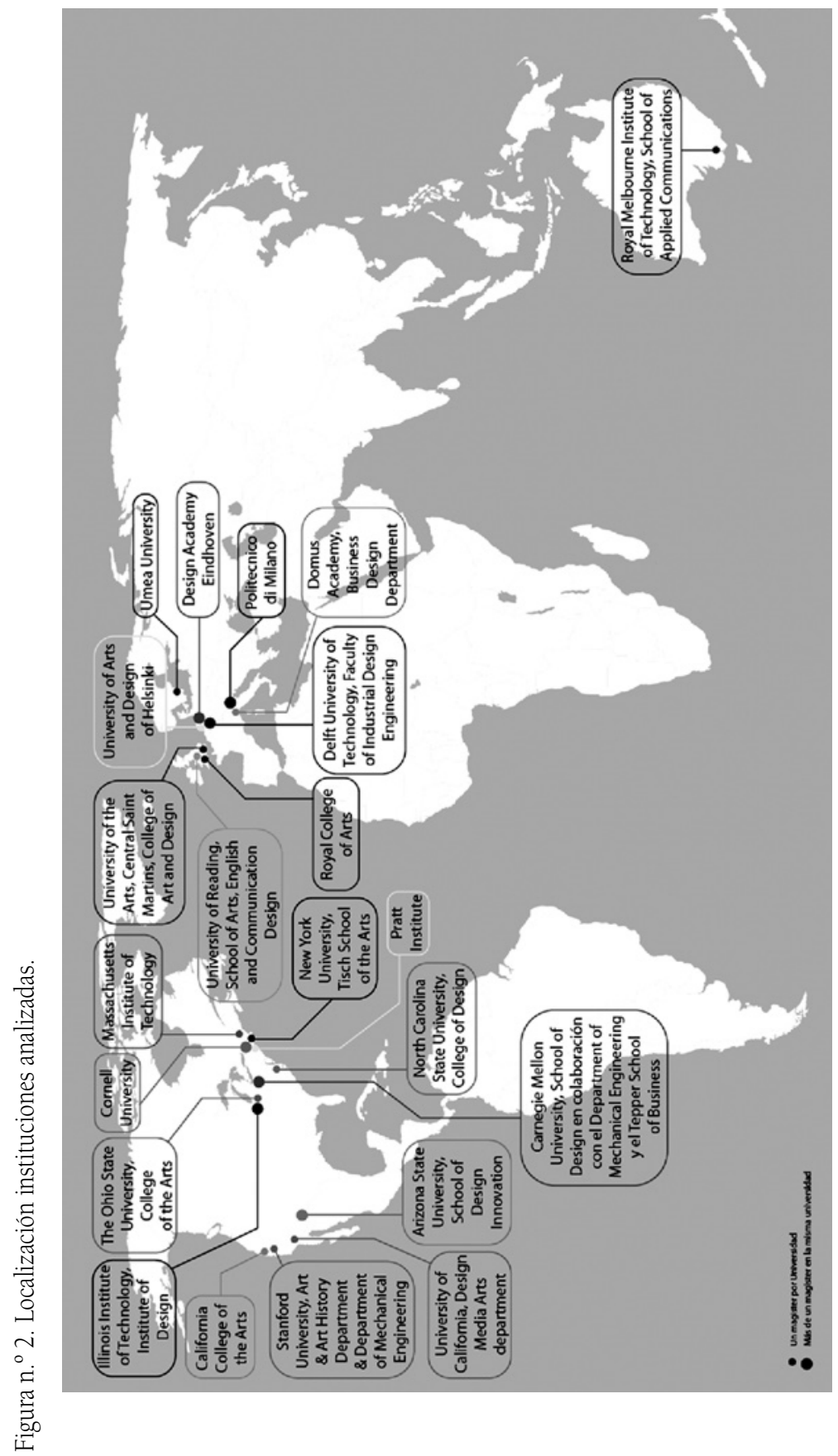




\section{Análisis de resultados}

En primer lugar, es posible señalar que existe un amplio espectro de áreas y enfoques cubiertos por los programas de posgrado en diseño, desde los que están ligados a una visión más artística de la disciplina, hasta aquellos programas más orientados a las necesidades de la industria. Dentro de estos últimos, existe una tendencia importante hacia el diseño centrado en el usuario o el User-centered Design y hacia el aporte del diseño en mejorar tanto la productividad de la empresa, como la calidad de vida de las personas.

De acuerdo con el primer listado de programas analizados (98 en total), se confirma que tanto el Diseño Industrial como la comunicación visual son las especialidades que agrupan mayor cantidad de programas, con un $27 \%$ y un $20 \%$ de las opciones, respectivamente (ver gráfico n. ${ }^{\circ} 1$ ).

Gráfico n. ${ }^{\circ}$ 1. Distribución de 98 programas de magíster en Diseño según macroáreas de estudio.

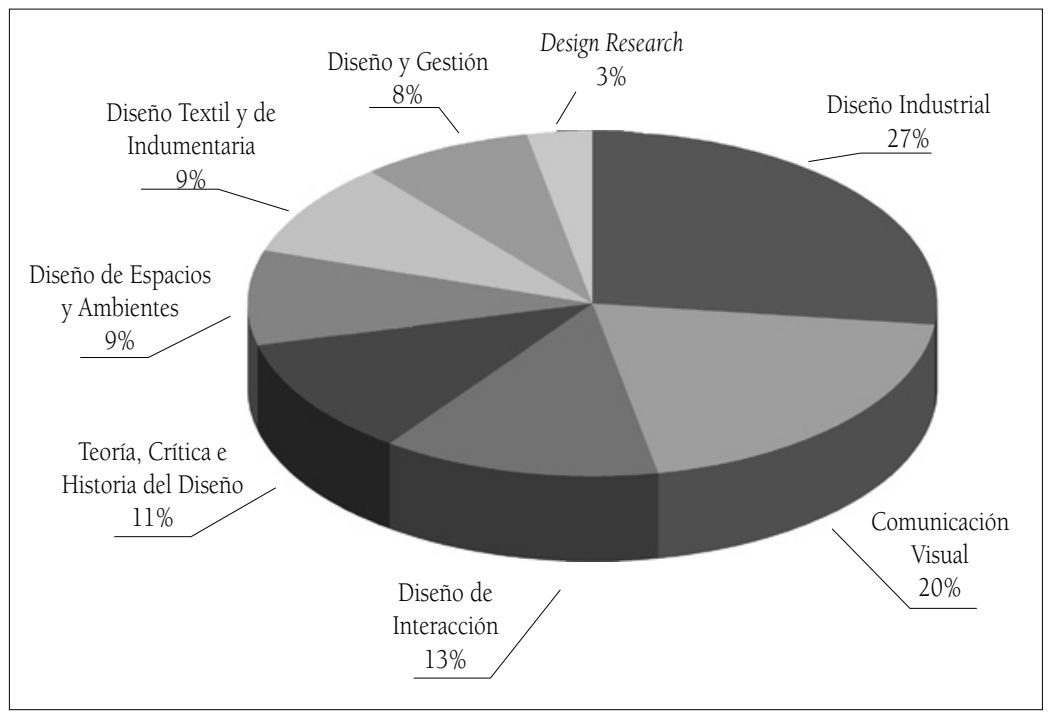

De acuerdo con el segundo filtro de programas analizados (39 en total), las áreas que agrupan mayor cantidad de programas son Diseño y Gestión y Diseño Industrial con un 32\% y un 29\%, respectivamente. Las siguen Diseño de Interacción (15\%), Comunicación Visual (12\%), 
Teoría, Crítica e Historia del Diseño (11\%), Espacios y Ambientes (6\%) y Design Research y Textil e Indumentaria (3\%) (ver gráfico n. ${ }^{\circ} 2$ ).

Gráfico n. ${ }^{\circ}$ 2. Distribución de 39 programas de magíster en Diseño según macroáreas de estudio.

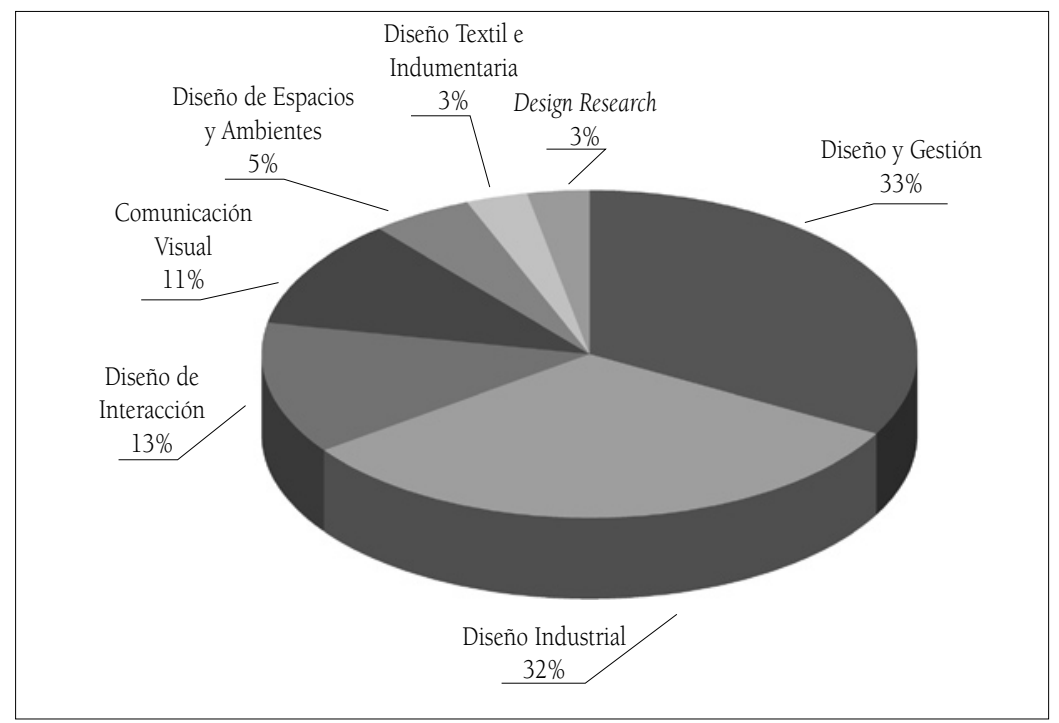

Los primeros programas de magíster en diseño surgen en la década de los $60^{6}$, coincidiendo con los inicios de la profesión del diseñador y de la relación del diseño con las ciencias. Es justamente en esta década cuando se funda la Design Research Society (DRS), organización cuyo objetivo es promover el estudio y la investigación en el proceso de diseño en todos sus campos. En 1962 se lleva a cabo en Inglaterra la "Conferencia en Metodologías del Diseño", evento que constituye la primera aproximación científica a las metodologías de diseño ${ }^{7}$. A esta conferencia le siguen otros encuentros de carácter

6 Es importante señalar que sólo se pudo obtener el año de origen de 23 programas de magíster. De éstos, los primeros programas de magíster en Diseño detectados son: Master in Apparel Design (Cornell University College of Fiber Science E Apparel Design, 1960), Joint Program in Design (Stanford University, Art \& Art History Department / Department of Mechanical Engineering, 1965), Design Management and Planning (The Ohio State University, College of the Arts, 1968), Master in Design: Interior Design (Cornell University, 1969) y Master of Science in Human Environment Relations (Cornell University, 1969).

7 Ver Bayazit (2004). 
internacional ${ }^{8}$ y una serie de publicaciones como Archer (1965) y Gregory (1966). Tal como afirma Bayazit (2004), la década de los 90 marcará un periodo de expansión de la profesión, a través de la incorporación de departamentos de diseño en múltiples empresas, el surgimiento de políticas de promoción, apoyo y financiamiento del diseño por parte del sector público y la proliferación de publicaciones, tales como Design Journal, Journal of Design Research y CoDesign, entre otras.

Es interesante señalar que 12 del total de 39 programas analizados fueron creados en los últimos diez años. De éstos, la mayoría pertenece al ámbito de Diseño y Gestión, manifestando una clara tendencia a incorporar este tipo de temáticas en la formación de diseño.

De los 39 programas analizados, sólo el Master of Design in Graphic Communication (Royal Melbourne Institute of Technology) se dicta en formato online. Este programa, perteneciente al área de Diseño y Gestión, exige a los postulantes experiencia laboral en diseño y un primer grado en una disciplina relacionada o reconocida como equivalente. Con un claro enfoque hacia la formación de product managers, este programa entrega competencias que vinculan el quehacer del diseñador con la gerencia de negocios.

Al realizar un análisis de contenido de los sitios web de cada uno de los programas, es posible constatar interesantes resultados en cuanto a los diversos enfoques y posturas frente a la disciplina del diseño (ver gráfico n. ${ }^{\circ}$ 3). Destacan las palabras Producto y Proyecto con 173 y 88 menciones respectivamente, seguidas por Tecnologías (72), Desarrollo, Industrial e Investigación (66), e Interacción (59). Esto podría sugerir que en la enseñanza de posgrado en diseño, la actividad proyectual asociada al desarrollo de nuevas tecnologías es central. Tanto en los programas de carácter académico como en los programas con un énfasis más profesional, se hace referencia a la investigación (66), estrategias (38) y metodologías (31) aplicadas al proyecto.

8 'The Design Method' in Birmingham, 1965 (Gregory, 1966), and 'Design Methods in Architecture', in Portsmouth, 1967 (Broadbent and Ward, 1969). 
Gráfico n. ${ }^{\circ}$ 3. Análisis de contenido de sitios web de los 39 programas seleccionados (palabras más frecuentes).

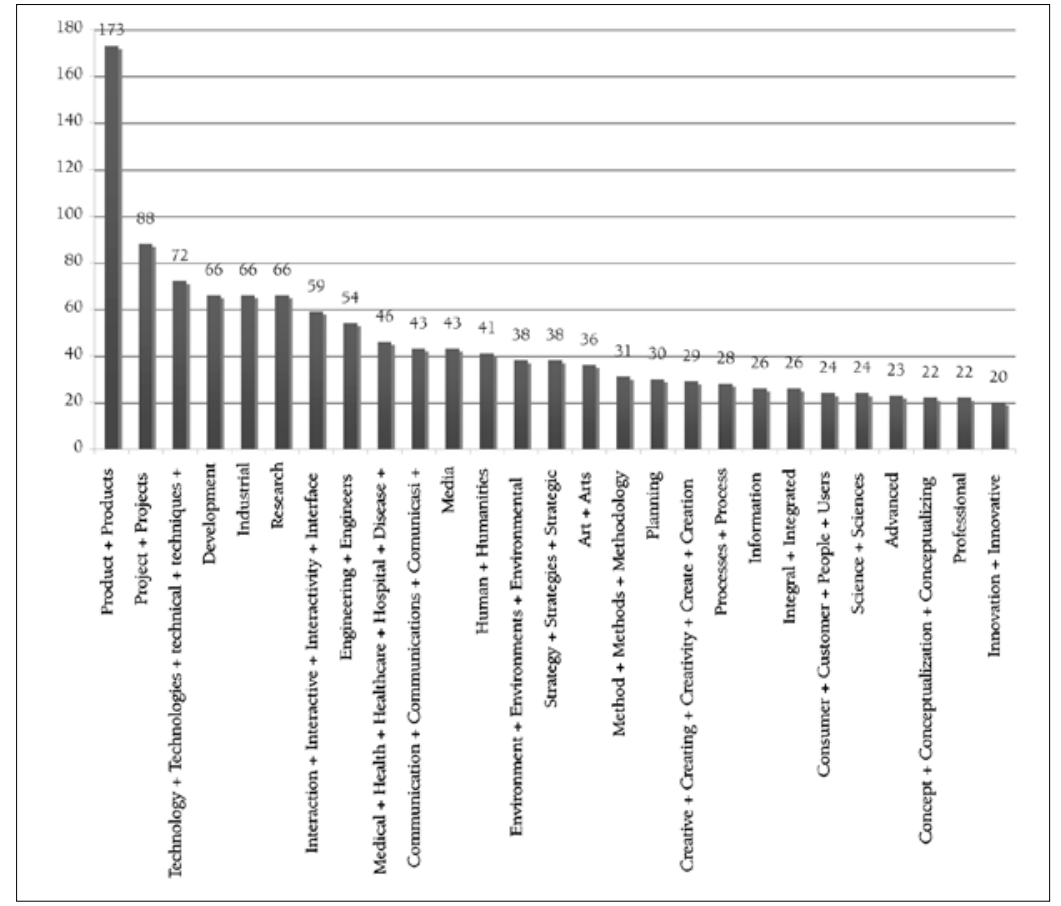

Destaca también la alta relación del diseño con la ingeniería (54 menciones) (la cual es bastante estrecha en el campo del diseño industrial) y con temas del ámbito de la salud (46 menciones). Esta última tendencia se refleja en el Master of Science Integrated Product Design: Medisign (Delft University of Technology) ${ }^{9}$, el cual pone énfasis en el desarrollo de productos para las personas que se desempeñan en el ámbito de la salud, basándose en la premisa de que ésta es una de las áreas más dinámicas tanto del mundo desarrollado (énfasis en la prevención) como en desarrollo (énfasis en sanación y cuidado). Las áreas de profundización de este programa son: Diseño para el Diagnóstico, Diseño para el Tratamiento, Diseño para la Sala de Operaciones, Productos, Cuidado y Diseño para Discapacitados.

9 Este programa constituye una especialización del Master Program Integrated Product Design y Design for Interaction de la Delft University of Technology. 
El Master of Design del Illinois Institute of Technology (Chicago) tiene un enfoque integral del diseño, sin embargo, también incorpora temas relacionados con el ámbito de la salud. Dentro de los cursos de especialización ofrecidos, se encuentran Designing ACCESS Healthcare y Special Problems: Re-imaging Healthcare. A su vez, tanto el Master of Science in Human Environment Relations (Cornell University) como el Master in Design: Interior Design (Cornell University) incluyen el campo de la salud dentro de los diversos ámbitos de acción del diseñador en sus respectivas especialidades.

Por otro lado, la preponderancia de los términos relacionados a las palabras interacción (59 menciones), humano (41 menciones) y usuario (24), reflejan una clara preocupación por parte de la disciplina del diseño en hacer un aporte en mejorar la calidad de vida de las personas. En este sentido, destaca el Master in Humanitarian Design and Sustainable Living (Design Academy Eindhoven, Holanda), el cual plantea una perspectiva humanitaria del diseño que pueda llevar a la creación de nuevos espacios y a un nuevo sentido de pertenencia al mundo.

\section{Principales tendencias en los programas de magíster en diseño seleccionados}

\section{1. Énfasis en la práctica y el vínculo con la realidad}

La enseñanza del diseño a nivel de posgrado muestra una importante tendencia hacia el campo profesional, ya que gran parte de los programas se basan en su vinculación con el medio externo, ya sea en el ámbito empresarial, cultural, educacional, social o productivo. En efecto, 20 de los 39 programas analizados se definen explícitamente como programas de carácter profesionalizante, con énfasis en la vinculación de la disciplina con el contexto social, productivo o empresarial. Independientemente del área temática, dichos programas colaboran en forma directa con empresas asociadas, ya sea para el desarrollo de proyectos de taller, aulas o laboratorios, proyectos de tesis o para prácticas profesionales (ver tabla n. ${ }^{\circ} 1$ ). Esto es especialmente relevante en los programas del área Diseño y Gestión, muchos de los cuales vinculan al diseño con empresas. En la mayoría de estos casos, 


\section{la actividad de graduación consiste en un proyecto final vinculado a una empresa, más que una tesis de carácter académico.}

Tabla n. ${ }^{\circ}$. Programas de magíster que explicitan su vínculo profesional.

\begin{tabular}{|c|c|c|c|}
\hline Programa & Área & Vínculos con empresas & $\begin{array}{l}\text { Actividad de } \\
\text { graduación }\end{array}$ \\
\hline $\begin{array}{l}\text { Communication. Royal } \\
\text { Master of Design in } \\
\text { Graphic Melbourne } \\
\text { Institute of Technology, } \\
\text { School of Applied } \\
\text { Communications, } \\
\text { Australia } \\
\end{array}$ & $\begin{array}{l}\text { Diseño y } \\
\text { Gestión }\end{array}$ & $\begin{array}{l}\text { Programa que permite al graduado pasar a } \\
\text { las posiciones avanzadas de gestión, diseño } \\
\text { de liderazgo y como product manager. } \\
\text { Fuerte vínculo con la industria. Programa } \\
\text { que sirve de nexo entre el diseñador y el } \\
\text { gerente de negocios en empresas de diseño }\end{array}$ & No se señala \\
\hline $\begin{array}{l}\text { Creative Business } \\
\text { Management, University } \\
\text { of Arts and Design of } \\
\text { Helsinki, Finlandia }\end{array}$ & $\begin{array}{l}\text { Diseño y } \\
\text { Gestión }\end{array}$ & $\begin{array}{l}\text { Conexiones con empresas: los graduados } \\
\text { pueden trabajar como profesionales de } \\
\text { la producción de los medios, artistas } \\
\text { del medio, productores, diseñadores e } \\
\text { investigadores (capaces de formar su propia } \\
\text { empresa) }\end{array}$ & Tesis final \\
\hline $\begin{array}{l}\text { MBA in Design Strategy. } \\
\text { California College of the } \\
\text { Arts, Estados Unidos }\end{array}$ & $\begin{array}{l}\text { Diseño y } \\
\text { Gestión }\end{array}$ & $\begin{array}{l}\text { Programa con carácter profesional y } \\
\text { vínculos con empresas }\end{array}$ & $\begin{array}{l}\text { Proyecto de Taller } \\
\text { (Venture Studio) }\end{array}$ \\
\hline \begin{tabular}{|l|} 
Master in Business \\
Design. Domus Academy, \\
Business Design \\
Department, Italia
\end{tabular} & $\begin{array}{l}\text { Diseño y } \\
\text { Gestión }\end{array}$ & $\begin{array}{l}\text { Asociación con empresas como 3M - } \\
\text { Autogrill - Consorzio del Franciacorta } \\
\text { - Deborah - Distretto del tessile di Lecco } \\
\text { - Ducati Motors - Elica - Fondazione Valore } \\
\text { Italia - Moleskine - Moreschi - Perini Navi } \\
\text { - Pininfarina Extra - Piquadro - Slow Food - } \\
\text { Unicredit Banca d'Impresa - Unilever }\end{array}$ & $\begin{array}{l}\text { Tesis relacionada con } \\
\text { una empresa }\end{array}$ \\
\hline $\begin{array}{l}\text { Industrial Design } \\
\text { Engineering. Royal } \\
\text { College of Arts, } \\
\text { Inglaterra } \\
\end{array}$ & $\begin{array}{l}\text { Diseño y } \\
\text { Gestión }\end{array}$ & $\begin{array}{l}\text { Asociación con empresas como Sony, } \\
\text { Unilever, Proctor \& Gamble, B\&Q, Dyson, } \\
\text { Targetti Lighting, BTG y Bank of America }\end{array}$ & $\begin{array}{l}\text { Práctica profesional } \\
+ \text { disertación del área } \\
\text { Historia y Crítica }\end{array}$ \\
\hline $\begin{array}{l}\text { Innovation Management, } \\
\text { University of the Arts, } \\
\text { Central Saint Martins, } \\
\text { College of Art and } \\
\text { Design, Inglaterra }\end{array}$ & $\begin{array}{l}\text { Diseño y } \\
\text { Gestión }\end{array}$ & $\begin{array}{l}\text { Asociación con empresas como Proctor } \\
\text { and Gamble, LG Electronics, Hyundai } \\
\text { Motors, Panasonic, Nokia, Design Council, } \\
\text { Industrial Design Society of America, Hong } \\
\text { Kong Design Centre, Design Management } \\
\text { Institute, Royal Society of Arts, Korean } \\
\text { Institute of Design Promotion }\end{array}$ & \begin{tabular}{|l|} 
Proyecto de \\
colaboración: \\
sostenida y compleja \\
investigación que le \\
permite explorar y \\
localizar la práctica \\
dentro de la zona \\
de gestión de \\
innovación \\
\end{tabular} \\
\hline \begin{tabular}{|l|} 
Master of Strategic \\
Product Design. Delft \\
University of Technology, \\
Faculty of Industrial \\
Design Engineering, \\
Holanda
\end{tabular} & $\begin{array}{l}\text { Diseño y } \\
\text { Gestión }\end{array}$ & $\begin{array}{l}\text { Programa con carácter profesional y } \\
\text { vínculos con empresas }\end{array}$ & Proyecto final \\
\hline
\end{tabular}




\begin{tabular}{|c|c|c|c|}
\hline $\begin{array}{l}\text { Master in Strategic } \\
\text { Design. Design of } \\
\text { the Value Offering. } \\
\text { Politecnico di Milano, } \\
\text { Italia }\end{array}$ & \begin{tabular}{|l} 
Diseño y \\
Gestión
\end{tabular} & $\begin{array}{l}\text { Asociación con empresas como 3M, Alessi, } \\
\text { Artemide, Artsana, Benetton, Bticino, } \\
\text { Domino, DUNI, Gemeaz-Ticket Restaurant, } \\
\text { Gewiss, Illy Caffê, Italtel, LOréal, Luceplan, } \\
\text { MH-Way, Ocean, Philips, Piaggio, Pirelli, } \\
\text { Qfree ASA, Lorenz S.p.A, Plastitex S.p.A., } \\
\text { Nardi S.p.A, Sofar Sonear, Trend Group } \\
\text { S.p.A, Panasonic Italia S.p.A. }\end{array}$ & $\begin{array}{l}\text { Proyecto de tres } \\
\text { meses: diseño } \\
\text { estratégico de } \\
\text { proyectos dentro } \\
\text { de una empresa } \\
\text { concreta }\end{array}$ \\
\hline $\begin{array}{l}\text { MA Information Design. } \\
\text { University of Reading, } \\
\text { School of Arts, English } \\
\text { and Communication } \\
\text { Design, Inglaterra }\end{array}$ & $\begin{array}{l}\text { Comunicación } \\
\text { visual }\end{array}$ & $\begin{array}{l}\text { Programa con carácter profesional y } \\
\text { vínculos con empresas }\end{array}$ & Tesis \\
\hline $\begin{array}{l}\text { MA Typeface Design. } \\
\text { University of Reading, } \\
\text { School of Arts, English } \\
\text { and Communication } \\
\text { Design, Inglaterra }\end{array}$ & $\begin{array}{l}\text { Comunicación } \\
\text { visual }\end{array}$ & $\begin{array}{l}\text { Programa con carácter profesional y } \\
\text { vínculos con empresas }\end{array}$ & $\begin{array}{l}\text { Desarrollo de una } \\
\text { tipografía y sus } \\
\text { variantes. Registro } \\
\text { del proceso. } \\
\text { Investigación } \\
\text { histórica y ensayo } \\
\text { sobre tipografía. }\end{array}$ \\
\hline \begin{tabular}{|l|} 
Master in Design: \\
Communication \\
Planning \& Information \\
Design. Carnegie Mellon \\
University, School of \\
Design
\end{tabular} & $\begin{array}{l}\text { Comunicación } \\
\text { visual }\end{array}$ & $\begin{array}{l}\text { Asociación con empresas como Motorola, } \\
\text { Microsoft, University of Pittsburgh Medical } \\
\text { Center, Carnegie Museum of Art, Samsung } \\
\text { Electronics y el U.S. Postal Service }\end{array}$ & \begin{tabular}{|l|} 
Tesis (dos semestres) \\
y proyecto final (dos \\
semestres)
\end{tabular} \\
\hline $\begin{array}{l}\text { Master of Design. Illinois } \\
\text { Institute of Technology, } \\
\text { Institute of Design, } \\
\text { Estados Unidos }\end{array}$ & Design Research & $\begin{array}{l}\text { Programa con carácter profesional y } \\
\text { vínculos con empresas }\end{array}$ & Proyecto \\
\hline \begin{tabular}{|l|} 
Interactive \\
Telecommunications \\
Program. New York \\
University, Tisch School \\
of the Arts, Estados \\
Unidos
\end{tabular} & $\begin{array}{l}\text { Diseño de } \\
\text { interacción }\end{array}$ & $\begin{array}{l}\text { Programa con carácter profesional y } \\
\text { vínculos con empresas }\end{array}$ & Tesis \\
\hline $\begin{array}{l}\text { Master of Science Design } \\
\text { for Interaction. Delft } \\
\text { University of Technology, } \\
\text { Faculty of Industrial } \\
\text { Design Engineering, } \\
\text { Holanda }\end{array}$ & $\begin{array}{l}\text { Diseño de } \\
\text { interacción }\end{array}$ & $\begin{array}{l}\text { Programa con carácter profesional y } \\
\text { vínculos con empresas }\end{array}$ & \begin{tabular}{|l|} 
Proyecto final, que \\
puede ser realizado \\
en conjunto con una \\
empresa o en alguno \\
de los centros de \\
investigación de la \\
Facultad de Diseño e \\
Ingeniería
\end{tabular} \\
\hline
\end{tabular}




\begin{tabular}{|c|c|c|c|}
\hline $\begin{array}{l}\text { Master of Product } \\
\text { Development. Carnegie } \\
\text { Mellon University, } \\
\text { School of Design en } \\
\text { colaboración con } \\
\text { el Department of } \\
\text { Mechanical Engineering } \\
\text { y el Tepper School } \\
\text { of Business, Estados } \\
\text { Unidos }\end{array}$ & $\begin{array}{l}\text { Diseño } \\
\text { industrial }\end{array}$ & $\begin{array}{l}\text { Asociación con empresas como Ford, } \\
\text { Respironics y BodyMedia }\end{array}$ & $\begin{array}{l}\text { Proyecto durante } \\
\text { el desarrollo del } \\
\text { magíster (no es una } \\
\text { actividad separada) }\end{array}$ \\
\hline $\begin{array}{l}\text { Joint Program in } \\
\text { Design. Stanford } \\
\text { University, Art \& Art } \\
\text { History Department } \\
\text { y el Department of } \\
\text { Mechanical Engineering, } \\
\text { Estados Unidos }\end{array}$ & $\begin{array}{l}\text { Diseño } \\
\text { industrial }\end{array}$ & $\begin{array}{l}\text { Asociación con empresas como Mozilla y } \\
\text { Global Living, entre otras }\end{array}$ & Proyecto final \\
\hline $\begin{array}{l}\text { Master in Advanced } \\
\text { Product Design. Umea } \\
\text { University, Suecia }\end{array}$ & $\begin{array}{l}\text { Diseño } \\
\text { industrial }\end{array}$ & $\begin{array}{l}\text { Programa con carácter profesional y } \\
\text { vínculos con empresas }\end{array}$ & Proyecto final \\
\hline $\begin{array}{l}\text { Master of Science } \\
\text { Integrated Product } \\
\text { Design. Delft University } \\
\text { of Technology, Faculty } \\
\text { of Industrial Design } \\
\text { Engineering, Holanda }\end{array}$ & $\begin{array}{l}\text { Diseño } \\
\text { industrial }\end{array}$ & $\begin{array}{l}\text { Programa con carácter profesional y } \\
\text { vínculos con empresas }\end{array}$ & $\begin{array}{l}\text { Proyecto final, que } \\
\text { puede ser realizado } \\
\text { en conjunto con una } \\
\text { empresa o en alguno } \\
\text { de los centros de } \\
\text { investigación de la } \\
\text { Facultad de Diseño e } \\
\text { Ingeniería }\end{array}$ \\
\hline $\begin{array}{l}\text { Master in Humanitarian } \\
\text { Design and Sustainable } \\
\text { Living. Design Academy } \\
\text { Eindhoven, Holanda }\end{array}$ & $\begin{array}{l}\text { Diseño } \\
\text { industrial }\end{array}$ & $\begin{array}{l}\text { Programa que combina lo teórico con lo } \\
\text { práctico }\end{array}$ & $\begin{array}{l}\text { Tesis + propuesta de } \\
\text { diseño }\end{array}$ \\
\hline $\begin{array}{l}\text { Master in Conceptual } \\
\text { Design in Context. } \\
\text { Design Academy } \\
\text { Eindhoven, Holanda }\end{array}$ & $\begin{array}{l}\text { Diseño } \\
\text { industrial }\end{array}$ & $\begin{array}{l}\text { Asociación con empresas como Unilever, } \\
\text { Sara Lee y Heinz }\end{array}$ & \\
\hline
\end{tabular}

A modo de ejemplo, el Master in Business Design (Domus Academy, Milán) plantea que el programa otorga un entorno adecuado para que los estudiantes "aprendan haciendo", en el cual el análisis de problemas y las soluciones de diseño permiten ser verificadas a través del trabajo con una red de empresarios y empresas que participan activa y directamente en la formación de los estudiantes.

En el mismo sentido, pero con otro enfoque, los programas de Master de la Design Academy Eindhoven (Holanda) ponen énfasis en la investigación aplicada al diseño, combinando distintas herramientas 
de investigación, desarrollo de proyectos y salidas a terreno en colaboración con artesanos, industrias e instituciones en todo el mundo (Kenia, Perú o India, por ejemplo).

\subsection{Multidisciplinariedad}

La multidisciplinariedad es otra característica transversal a los programas analizados y se manifiesta en prácticas colaborativas no sólo con alumnos que provienen de distintas formaciones académicas (artes, ciencias o ingeniería), sino también de los profesores que pertenecen a distintas facultades y ámbitos de expertise. Un total de 16 programas explicitan esta variable como un atributo del programa que facilita el intercambio de experiencias y conocimientos y permite al graduado desenvolverse en un ámbito profesional que aborda problemas de diseño cada vez más complejos. Por el contrario, sólo 10 programas presentan como requisito de ingreso una licenciatura o experiencia laboral en el ámbito del diseño.

La complementariedad con otras disciplinas se manifiesta también en la asociación de las escuelas y departamentos de diseño que otorgan el grado con otras instituciones pertenecientes a diversas disciplinas. Por ejemplo, dos programas se dictan bajo el formato de doble grado (ambos correspondientes al área Diseño y Gestión): (1) Industrial Design Engineering, MA + MSc Double Masters Joint Course (Royal College of Arts, Imperial College London) ${ }^{10}$, (2) Master of Design / Master of Business Administration (Illinois Institute of Technology). ${ }^{11}$

El primer programa entrega habilidades que se extienden más allá del Diseño industrial, abarcando la experimentación, la teoría y el espíritu empresarial. Este aprendizaje colaborativo se sustenta en las capacidades y culturas de dos organizaciones muy diferentes: una universidad predominantemente técnica (Imperial College de Londres)

10 Independientemente de su origen (artístico o técnico) los graduados reciben un MA (RCA) y una MSc más un diploma (ambos con el Imperial College London). A su vez, los alumnos deben elegir una de tres áreas de especialización, las que se consideran como fundamentales para el futuro diseño e innovación: Diseño Experimental, Diseño para la Fabricación y Empresa de Diseño.

11 Este programa tiene una duración de dos años más un trimestre para quienes tienen licenciatura en diseño y tres años más un trimestre para quienes no tienen licenciatura en diseño. 
y una de Arte y Diseño (RCA). El segundo programa combina la formación profesional de posgrado en diseño con el ámbito de los negocios y supone un hito importante en la coevolución del diseño, la gestión y la innovación. Plantea que el diseño se ha vuelto un elemento esencial en el ámbito empresarial, al promover un enfoque centrado en el usuario. De esta manera, el Master of Design/MBA entrega competencias referidas a métodos que permiten la innovación con énfasis en la gestión de la comercialización, la contabilidad del proyecto y la organización y comportamiento de los consumidores.

También se observan programas que, si bien no otorgan doble grado, sí se dictan en conjunto con otras escuelas o facultades. Por ejemplo, el Master of Science Integrated Product Design: Medisign (Delft University of Technology), se dicta en cooperación con la Facultad de Medicina de Erasmus MC (Rotterdam). El Joint Program in Design (Stanford University) es impartido por los departamentos de Ingeniería Mecánica y el Departamento de Arte e Historia del Arte. Con una vasta experiencia, este programa se basa desde su origen en la relación entre diseño e ingeniería centrada en el usuario ${ }^{12}$. Por su parte, el MA in Creative Business Management (University of Arts and Design of Helsinki) es un programa nuevo (su primer año de existencia es el 2009) que se dicta en colaboración con la Facultad de Economía de Turku (Pori Unit) y que ofrece experiencia en el manejo multidisciplinario de los procesos creativos y la economía de las empresas. Este programa se centra en todo el ciclo de vida del producto, desde el concepto de diseño a su producción, el conocimiento y gestión de la marca y la gestión de procesos creativos e investigación de la producción inmaterial, siempre desde una perspectiva centrada en el usuario.

Algunos programas intentan nivelar aquellos alumnos que no provienen del ámbito del diseño, con la finalidad de prepararlos para las etapas posteriores. Por ejemplo, el Master of Industrial Design (North Carolina State University) establece que el primer año del programa

12 El Joint Program in Design de Stanford University fue creado en 1958. El JPD tiene una duración de dos años y la opción de graduarse con un MS en Ingeniería con mención en Diseño de Productos, entregado por el Departamento de Ingeniería Mecánica o un MFA entregado por el Departamento de Arte e Historia del Arte. En este caso, el alumno puede elegir graduarse con el título que se ajuste de mejor manera a su formación de pregrado y a sus expectativas laborales. 
entrega una base sólida de formación en principios formales de diseño para los alumnos que no tienen una formación previa en el área. Un proceso similar se aplica en Chile con el programa College PUC.

\subsection{El usuario como centro del proyecto de diseño}

Otro aspecto transversal a gran parte de los programas analizados, independientemente del área del diseño al que pertenezcan, es el enfoque hacia un diseño centrado en el usuario, para lo cual se requiere de la aplicación de herramientas de investigación de carácter cualitativo y etnográfico como el punto de partida del proyecto. Así, gran parte de los programas plantean que la definición del problema y el proceso de diseño son tan importantes como el producto final, por lo que prácticamente todas las mallas curriculares incluyen cursos sobre metodologías de investigación social, así como herramientas de análisis para informar correctamente las decisiones de diseño que se tomen en etapas posteriores del proceso.

El MA Information Design (University of Reading), por ejemplo, está enfocado en la comunicación visual centrada en el usuario, en el cual el alumno desarrolla sus habilidades prácticas a través de proyectos que involucran el desarrollo de material complejo para diferentes tipos de usuarios. En el mismo sentido, el Master in Design: Interior Design (Cornell University) se fundamenta en la premisa de que los usuarios de un espacio físico determinado son diversos y tienen diferentes necesidades o características individuales (género, etapa del ciclo de vida y estructura familiar), así como organizacionales (cultura de la organización, metas y estructura) que afectan nuestras necesidades con respecto al entorno y determinan su uso. Por lo tanto, se plantea que el entendimiento de las necesidades de la organización y del usuario son tan relevantes como los aspectos financieros, tecnológicos y formales del diseño.

Por su parte, el Master of Science Design for Interaction (Delft University of Technology) enfatiza el estudio, conceptualización, creación de prototipos y testeo de interacción usuario-producto. Los estudiantes aprenden cómo derivar el conocimiento especializado en ciencias del comportamiento humano hacia parámetros de diseño, 
usando estos conceptos en el desarrollo de nuevos productos. Para esto, deben aprobar cursos como Comprensión de Producto, Uso y Experiencia, Contexto y Conceptualización, Investigación Observacional, Diseño de Tecnologías Interactivas y Diseño de Comunicación Visual; conocimientos que luego son aplicados a dos proyectos de taller.

De la misma manera, en el Master in Design Research (IIT) los alumnos pueden construir su propio currículum, una vez aprobados los cursos mínimos referidos a métodos de observación y comprensión del usuario en contextos específicos, análisis de información compleja, desarrollo y exploración de soluciones alternativas y prototipaje de futuras innovaciones y análisis. ${ }^{13}$

\subsection{Importancia de la investigación aplicada al proyecto de diseño}

Consecuentemente con lo planteado en el punto anterior, gran parte de los programas plantean que la definición del problema y el proceso de diseño son tan importantes como el producto final, por lo que prácticamente todas las mallas curriculares también incluyen cursos que entregan competencias de investigación de carácter cualitativo y etnográfico, tales como investigación de mercado, métodos de observación, comprensión del usuario, comportamientos del consumidor, análisis de información compleja y desarrollo y exploración de soluciones innovadoras. En otras palabras, si bien gran parte de los programas son de carácter profesionalizante, la investigación centrada en el usuario constituye una herramienta de análisis fundamental para informar correctamente las decisiones de diseño que se tomen en todas las etapas del proceso del diseño. El programa de Advanced Product Design (Umea University) tiene como objetivo identificar y definir el problema como punto de partida del proceso de diseño. Partiendo desde la innovación como base para la definición del encargo de diseño, la Universidad de Umea plantea distintas fases en el desarrollo del proyecto, siendo la investigación

13 Ejemplos de las áreas de especialización incluyen comunicación visual, diseño de productos, planificación estratégica, investigación de usuario, métodos de investigación en diseño y diseño de sistemas. 
la segunda de ellas y de gran relevancia para informar el problema de diseño.

Por su parte, el Master in Business Design (Domus Academy) entrega las competencias necesarias para la investigación y la apertura hacia nuevos negocios que entren en el modelo clásico de la empresa, a partir de la identificación de oportunidades y estrategias de mercado innovadoras, la investigación del modelo de organización y las características del "made in Italy", ganando así la capacidad de comprender, profundizar y optimizar el éxito de la empresa y sus procesos, manteniendo una visión que no sólo aproveche mejor el valor de la marca, sino que también comprenda el mercado y su sector. Tendencia importante al considerar que el valor de un producto no necesariamente radica en el lugar donde se manufactura, sino más bien donde se idea o proyecta.

Aquellos programas que son de carácter más bien académico, con énfasis en la investigación científica, plantean un enfoque similar. El Master of Science in Design: Visual Communication Design (Arizona State University), programa dedicado fundamentalmente a la investigación, se centra en el estudio sistemático y metodológico de temas relevantes a la práctica del diseño. Por su parte, el objetivo del MFA Degree in Design I Media Arts (University of California) es que el alumno realice un proyecto que incorpore la investigación y exploración teórica sobre un tema, para lo cual durante el primer año del programa, los alumnos deben tomar ramos que los exponen a nuevas formas de pensar y hacer, sumándolo a sus propias experiencias y áreas de especialización.

También el Master of Science in Design: New Product Innovation (Arizona State University) pone el énfasis en la investigación y se centra en el estudio sistemático y metodológico de temas relevantes a la práctica del diseño. Entre otros objetivos, plantea el desarrollo de habilidades de investigación especializada que apoyen el trabajo profesional de diseñadores industriales, diseñadores de interiores y comunicadores visuales, así como permitir que los profesionales del diseño adquieran las habilidades de investigación necesarias para su desempeño, tanto en carreras académicas como profesionales. Este 
programa de investigación basada en el proyecto provee a los alumnos con los conocimientos avanzados necesarios para tener un desempeño exitoso en el mundo del desarrollo de productos, para lo cual se apoya en cursos como Research Methods y Qualitative Research in Design, Video Etnography e Innovation and the Individual, entre otros.

Los programas de Master de la Design Academy de Eindhoven (Master in Humanitarian Design and Sustainable Living y Master in Conceptual Design in Context) también ponen el foco en la investigación y el análisis, para lo cual se espera que los alumnos no sólo desarrollen habilidades para diseñar, sino que también desarrollen habilidades intelectuales y creativas, con el fin de conducir y visualizar una investigación independiente de valor para otros profesionales. Con esta finalidad, durante el primer año cada estudiante investiga tres temas, tanto a nivel teórico como práctico, hacia una visualización de la investigación y su resultado final. El Master in Conceptual Design in Context plantea que los diseñadores necesitan estar conscientes de estos cambios y saber cuál es su rol en cada nivel, relacionando las necesidades locales, recursos tradicionales, tecnologías y mercados.

\subsection{El rol del diseño en la estrategia y gestión de empresas}

El enfoque centrado en el usuario se hace más relevante aun en los programas del área de Diseño y Gestión, lo cual es parte del creciente rol del diseño en la creación y gestión estratégica de las empresas. Estos programas destacan la necesidad de "conducir las organizaciones desde la perspectiva única del pensamiento del diseño"14 y de "generar las condiciones que permiten a los alumnos mirar el panorama de negocios actual desde una perspectiva sistemática de $360^{\circ}$, necesaria para el desarrollo de la innovación radical en productos, servicios y estrategias de comunicación"15. Así, el aporte del diseño a una mejor gestión de negocios se hace fundamental, tal como plantea el MBA In Design Strategy (California College of Arts): "Al unir los estudios de diseño, las finanzas, la estrategia, y la sustentabilidad, proporcionamos las herramientas para la gestión en los actuales mercados interconectados, con una visión de negocio sustentable,

14 MBA in Design Strategy (California College of Arts).

15 Master of ICT and Design for Innovation (Politecnico di Milano). 
significativa, ética, rentable, y realmente innovadora". En este programa, se busca que los estudiantes desarrollen soluciones para una serie de desafíos económicos y sociales, utilizando tanto técnicas de diseño, tales como investigación centrada en el cliente, elaboración de prototipos, crítica e iteración, como de la gestión del negocio. En este programa, las soluciones son evaluadas de acuerdo con qué tan bien los alumnos descubren a los usuarios y las necesidades del mercado, así como su valor de organización.

De la misma manera, el Master of Design / MBA (Illinois Institute of Technology), programa de doble grado cuyos métodos apuntan a la innovación a partir del diseño centrado en el usuario (organización, comportamiento) y de la gestión del proyecto (comercialización, contabilidad). Algunos de los cursos requeridos por el programa y que se enfocan en una mejor comprensión del usuario son: Cognitive Human Factors, Cultural Human Factors, Observing Users, Physical Human Factors, y Social Human Factors, entre otros. Todos estos cursos también pertenecen al programa de Master in Design de la misma universidad. En el MA in Creative Business Management (University of Arts and Design of Helsinki), los estudios se centran en diversos temas de producción, desde el concepto de diseño a la producción, el conocimiento y gestión de la marca y la gestión de procesos creativos e investigación de la producción inmaterial, siempre desde una perspectiva centrada en el usuario.

Por último, el Master in Strategic Design, Design of the Value Offering (Politecnico di Milano) define diseño estratégico como la unión entre el diseño y la estrategia de empresa, el cual se sustenta en las nuevas oportunidades que el diseño presenta para operar ante la creciente demanda social por nuevas generaciones de productos y servicios, coherentes con las presentes transformaciones socioculturales y desarrollo sustentable.

\section{Conclusiones}

A partir del análisis realizado, es posible confirmar que la creciente complejización del proyecto de diseño está siendo abordada por los programas de magíster en las escuelas seleccionadas, desde sus 
respectivas especialidades y perspectivas de la disciplina y la profesión. El énfasis en la práctica, la multidisciplinariedad, el usuario como centro del proyecto, la importancia de la investigación aplicada al diseño y el rol del diseño en la gestión de empresas, constituyen las principales tendencias de dichos programas, las cuales dan cuenta de una profesión que se encuentra cada vez más inserta en áreas estratégicas de la economía.

La acelerada creación y renovación de programas de magíster ha permitido perfilar y perfeccionar el enfoque y el perfil de egreso de sus alumnos. En el contexto nacional, hace tan sólo un par de décadas, un diseñador con grado de magíster se perfilaba como un profesional dedicado al desarrollo de la investigación y la docencia universitaria. El costo de este perfeccionamiento era asumido preferentemente por una institución universitaria y en algunos casos compartido con fondos públicos. A nivel internacional, empresas productivas como Coca-Cola, Disney, Ford o Airbus financian el perfeccionamiento de sus profesionales, además de trabajar colaborativamente en la creación de dichos programas, con la finalidad de formar profesionales idóneos para las necesidades del medio productivo real. Esta participación activa y directa del mundo empresarial asegura una mejor empleabilidad de los diseñadores, a partir de la vinculación anticipada generada por los proyectos colaborativos.

Por otro lado, es posible señalar que a las tradicionales especialidades del Diseño Industrial y comunicación visual se ha sumado con fuerza la variable gestión y diseño, confirmando con esto la tendencia creciente del mercado en demandar dichas competencias en los diseñadores profesionales, capacidades que les han permitido ocupar nuevos roles estratégicos al interior de las empresas. Hace no más de una década, el diseñador se desempeñaba como un profesional que comúnmente prestaba sus servicios desde afuera de la empresa, sin ninguna vinculación a las directrices estratégicas del directorio, ni a las condicionantes productivas. Esto retardaba y hacía menos concurrente el proceso de desarrollo de nuevos productos al interior de la empresa. 
Recientemente, conceptos asociados a perfiles de responsabilidad profesional, como el product manager o el gerente de gestión de productos, han aproximado al diseñador con los responsables comerciales de la marca. Hoy podemos apreciar que el diseñador, como actor fundamentalmente creativo, ha ido paulatinamente ocupando estos puestos estratégicos por sobre otros profesionales, ligados mayoritariamente al mundo del marketing y de las finanzas. Esto se genera en gran parte porque los niveles de confidencialidad y seguridad exigen que el diseño, como recurso estratégico diferenciador, sea parte del origen del producto y no de una variable de carácter simplemente estético que entra al final del proceso.

Tal como es posible de visualizar en esta investigación, gran parte de los programas de posgrado universitarios creados en los últimos diez años pertenecen al ámbito de diseño y gestión estratégica, la interacción y el design research. Al constituirse estos como ámbitos de acción multidisciplinar, en los cuales la acción del diseñador se traslapa con otras profesiones, es posible afirmar que la profesión del diseñador tiende a adaptarse constantemente tanto a la evolución de las economías donde se inserta, como a los cambios sociales. ${ }^{16}$

A partir del presente análisis, cabe preguntarse entonces cuál es el programa más pertinente a la realidad geográfica y económica de Chile, la cual, en gran parte, se basa en la exportación de commodities y donde el diseño de manufacturas y servicios podría constituir uno de los principales campos de acción tanto en el ámbito nacional como internacional. A su vez, un magíster en diseño en Chile debe plantearse como un programa de interés para otros países de Latinoamérica, región que presenta una escasa oferta de posgrados en diseño con las características presentadas en esta investigación.

En este contexto, es posible destacar el caso de Holanda, país pequeño pero con un alto nivel de industrialización, en el cual surgen propuestas interesantes como el Master in Humanitarian Design and Sustainable Living, el cual propone una clara tendencia hacia las necesidades socioculturales, geopolíticas, de sustentabilidad, 
de rescate de la artesanía, nuevas oportunidades industriales y tecnológicas, economía y valores humanos, como parte integral de una perspectiva humanitaria del diseño. Esta propuesta señala también una clara tendencia en cuanto a posicionar a la persona como centro del proyecto, respuesta que surge como una reacción al desequilibrio provocado por un agotado sistema económico global.

En este sentido, otra tendencia que surge desde el análisis de los posgrados en diseño es que el factor tecnológico ha dejado de constituirse como un elemento diferenciador. Es indudable que esta variable ha influido fuertemente en la enseñanza y la práctica del diseño a través del surgimiento de nuevos software y hardware que han acortado el proceso de desarrollo e implementación de nuevos productos y servicios. Sin embargo, paradójicamente esta variable no se muestra como relevante al momento de presentar un programa de magíster en diseño. Así, surgen otras directrices como más relevantes, las que podrían considerarse eventualmente como "variables blandas" de la disciplina. Tal es el caso de la interacción, la sustentabilidad y el mejoramiento de la calidad de vida, entre otras variables que se mezclan transversalmente en el contexto productivo, social e industrial. De esta manera, un nuevo magíster en diseño a nivel nacional, debiese abordar estas variables que responden a nuevos paradigmas del diseño.

Tal como plantea Gisele Raulik-Murphy, el apoyo, promoción y la educación en diseño constituyen los principales ejes para potenciar su uso como factor que otorga competitividad y agrega valor. Así, es posible diagnosticar diversos grados de impulso al diseño en las diversas economías del mundo. Mientras países como Canadá, Estados Unidos, Australia, Turquía, Dinamarca y Austria cuentan con programas públicos de promoción del diseño (para asistir a empresas de manera que lo usen como herramienta de mejoramiento del negocio); los gobiernos de México, Argentina, Brasil, Malasia y Sudáfrica, entre otros, apoyan a las empresas locales para que inviertan en diseño. Finalmente, países como la India, Finlandia, Japón, Nueva Zelanda, Corea del Sur, Holanda, República Checa y Eslovaquia cuentan con políticas públicas que incentivan la incorporación del diseño en el desarrollo productivo. ${ }^{17}$ 
Consecuentemente, es posible señalar que la investigación aquí presentada debe ampliarse hacia el análisis de programas de posgrado en diseño pertenecientes a países asiáticos, especialmente aquellos que cuentan con políticas de apoyo y promoción del diseño. Como economía emergente, Chile tiene una significativa relación con países como India y China, por lo que sería de interés verificar si las tendencias identificadas en este estudio se corroboran también en ellos. A su vez, a la luz de la creación de un magíster en Diseño en la PUC, se hace necesaria la investigación de programas de magíster en el contexto latinoamericano, de manera de comprender las tendencias presentes en los programas que constituyen una competencia más directa al nacional.

\section{Referencias bibliográficas}

Archer, L. B. (1965) Systematic Methods for Designers, London: The Design Research Council.

Bayasit, N. (2004) Investigating Design: A Review of Forty Years of Design Research. Design Issues: Volume 20, Number 1, winter 2004.

Broadbent, G. and Ward, A. (Eds.) (1969) Design Methods in Architecture, New York, NY: George Wittenborn Inc.

Gregory, S. A. (ed.) (1966) The Design Method, Butterworth, London.

Jones, J.C. (1984) Diseñar el Diseño, trad. por Iris Menéndez, Editorial G. Gili, Barcelona Santiago, Chile

Raulik-Murphy, G., Cawood, G., Larsen, P., Lewis, A. (2008) A comparative analysis of strategies for design in Finland and Brazil, University of Wales Institute Cardiff, Design Wales, UK, Proceedings of Design Research Society Biennial Conference, Sheffield, UK, 16-19, July 2008.

\section{Fuentes electrónicas}

http://www.design.cmu.edu/show_program.php?s=2\&t=1 Recuperado: 05/11/2009

https://design.asu.edu/msd/degree.shtml Recuperado: 28/05/09

http://www.rdg.ac.uk/typography/pg-taught/typ-pgtmainformationdesign.asp Recuperado: 06/05/2009

http://www.rdg.ac.uk/typography/pg-taught/typ-pgtmatypefacedesign.asp Recuperado: 06/05/2009 
http://www.human.cornell.edu/che/DEA/Academics/Graduate/MAID/index. cfm Recuperado: 28/05/09

http://www.human.cornell.edu/che/DEA/Academics/Graduate/MSHER/index. cfm Recuperado: 28/05/09

http://admissions.media.mit.edu/admissions/degrees-study Recuperado: 28/05/09

http://itp.nyu.edu/itp/program.php Recuperado: 06/04/2009

http://dma.ucla.edu/education/overview.php\#MFA Recuperado: 06/11/2009

http://www.io.tudelft.nl/live/pagina.jsp?id=b4c76e5e-3a59-4be9-a050c847d3a5fbb2\&lang=en Recuperado: 06/09/2009

http://www.design.cmu.edu/show_program.php?s=2\&t=3 Recuperado: 05/11/2009

http://ncsudesign.org/content/index.cfm/mode/l/fuseaction/page/filename/ master_industrialdesign.html Recuperado: 15/05/09

http://www-design.stanford.edu/PD/ Recuperado: 15/05/10

https://design.asu.edu/msd/degree.shtml Recuperado: 28/05/09

http://www.polidesign.net/mds/ Recuperado: 06/11/2009

http://www.design.polimi.it/new/pages.php?pagina=123 Recuperado: $17 / 6 / 09$

http://www.dh.umu.se/default.asp?ml=10454 Recuperado: 06/08/2009

http://www.io.tudelft.nl/live/pagina.jsp?id=d384ae3b-8c7c-4aa0-b76449c60abcc2c4\&lang=en Recuperado: 06/09/2009

http://www.io.tudelft.nl/live/pagina.jsp?id=d384ae3b-8c7c-4aa0-b76449c60abcc2c4\&lang=en Recuperado: 06/09/2009

http://www.designacademy.nl/indexen.htm Recuperado: 06/11/2009

http://www.esdi.uerj.br/pos-graduacao/index.html Recuperado: 06/11/2009

http://www.human.cornell.edu/che/fsad/academics/apparel-design-graduateprograms.cfm Recuperado: 28/05/09

http://www.design.osu.edu/dept_grad.html Recuperado: 05/07/2009

http://www.pratt.edu/design_management\# Recuperado: 06/11/2009

http://www.id.iit.edu/99/ Recuperado: 05/11/2009

http://www.cca.edu/academics/graduate/design-mba Recuperado: 26/05/09

http://www.cca.edu/academics/graduate/design/dualdesign Recuperado: $26 / 05 / 09$ 
http://www.rmit.edu.au/browse;ID=MC128 Recuperado: 17/6/09

http://www.domusacademy.com/eng/8/index.php?tmp=8\&id=15 Recuperado: 06/05/2009

http://www.polidesign.net/mds/ Recuperado: 06/11/2009

http://www.taik.fi/content/view/300/269/lang,en/ Recuperado: 17/6/09

http://www.rca.ac.uk/Default.aspx?ContentID=159950\&GroupID=160473\& Contentwithinthissection\&More=1 Recuperado: 06/05/2009

http://www.csm.arts.ac.uk/courses/postgraduate/innovation-management. htm\# Recuperado: 06/05/2009

http://www.io.tudelft.nl/live/pagina.jsp?id=b4609d2c-d99e-4cc3-8ab98baf31633c0a\&lang=en Recuperado: 06/09/2009

Recibido: 11/122009

Aceptad: 04/11/2010 